\title{
Acoustic-gravity nonlinear structures
}

\author{
D. Jovanović ${ }^{1}$, L. Stenflo ${ }^{2}$, and P. K. Shukla ${ }^{3}$ \\ ${ }^{1}$ Institute of Physics, P.O. Box 57, Yu-11001 Belgrade, Yugoslavia \\ ${ }^{2}$ Department of Plasma Physics, Umeå University, S-90187 Umeå, Sweden \\ ${ }^{3}$ Institut für Theoretische Physik IV, Ruhr-Universität Bochum, D-44780 Bochum, Germany
}

Received: 14 September 2001 - Accepted: 7 December 2001

\begin{abstract}
A catalogue of nonlinear vortex structures associated with acoustic-gravity perturbations in the Earth's atmosphere is presented. Besides the previously known KelvinStewart cat's eyes, dipolar and tripolar structures, new solutions having the form of a row of counter-rotating vortices, and several weakly two-dimensional vortex chains are given. The existence conditions for these nonlinear structures are discussed with respect to the presence of inhomogeneities of the shear flows. The mode-coupling mechanism for the nonlinear generation of shear flows in the presence of linearly unstable acoustic-gravity waves, possibly also leading to intermittency and chaos, is presented.
\end{abstract}

\section{Introduction}

In recent years there has been a growing interest in the nonlinear effects associated with large amplitude acousticgravity perturbations in the Earth's atmosphere. These efforts were motivated by the need to obtain more accurate predictions of the dynamics of the atmosphere under various meteorological conditions, including different profiles of the pressure and density, as well as the presence of shear flows.

The most commonly used model equations for the twodimensional, $\partial / \partial y=0$, large amplitude acoustic-gravity phenomena have the form

$$
\begin{aligned}
& {\left[\frac{\partial}{\partial t}-\left(\boldsymbol{e}_{y} \times \nabla \psi\right) \cdot \nabla\right] \nabla^{2} \psi-\frac{1}{4 H^{2}} \frac{\partial \psi}{\partial t}} \\
& \quad=-\left(\boldsymbol{e}_{y} \times \nabla q_{0}\right) \cdot \nabla \chi,
\end{aligned}
$$

and

$$
\left[\frac{\partial}{\partial t}-\left(\boldsymbol{e}_{y} \times \nabla \psi\right) \cdot \nabla\right] \chi=0
$$

Correspondence to: D. Jovanović (djovanov@ phy.bg.ac.yu) where the notations are

$$
\begin{aligned}
& \psi=\frac{\Psi}{\rho_{0}}, \chi=\frac{p_{0}^{1 / \gamma}}{\rho}\left(\frac{1}{p_{0}^{1 / \gamma}} \frac{d p_{0}}{d z}\right)_{z=z_{c r}}, \\
& \frac{1}{4 H^{2}}=\rho_{0}^{1 / 2} \frac{d^{2} \rho_{0}^{-1 / 2}}{d z^{2}} \text { and } q_{0}=\frac{p_{0}^{1-1 / \gamma}}{\left[(d / d z) p_{0}^{1-1 / \gamma}\right]_{z=z_{c r}}} .
\end{aligned}
$$

Here, $\Psi$ is the stream function, defined by $\boldsymbol{v}=-\boldsymbol{e}_{y} \times$ $\nabla \Psi / \rho_{0}$, where $\boldsymbol{v}$ is the fluid velocity. As usual, $p$ and $\rho$ are the pressure and density, and $\gamma(\gamma \approx 1.4)$ is the adiabatic constant. The subscript " 0 " denotes the unperturbed quantities, and $z=z_{c r}$ is the critical vertical position that will be defined later. Equations (1) and (2) were derived (Stenflo, 1990; Stenflo and Stepanyants, 1995) for low frequency disturbances in an almost incompressible fluid, $\delta p / p_{0} \ll$ $\delta \rho / \rho_{0} \ll 1$, usually referred to as the anelastic regime. Although the derivation (Stenflo, 1990) was restricted to exponential density profiles and perturbations with relatively short wavelengths and small (but finite) amplitudes, the system above was used as a simple model to study certain large amplitude phenomena, such as self-organization into coherent structures. A more rigorous analysis (Jovanović et al., 2001) shows that the same equations are also valid in the strongly nonlinear regime, in the vicinity of the extremum $z=z_{c r}$ of the background density, where the condition $\left(\nabla^{2} \rho_{0} / \rho_{0}\right)^{1 / 2} \gg\left|\nabla \rho_{0} / \rho_{0}\right|$ is fulfilled.

In the nonlinear regime, the dynamics of the acousticgravity phenomena, as described by Eqs. (1) and (2), is dominated by nonlinear convection, and as a consequence, various coherent localized vortex structures may arise. The form of the vortices is determined by the spatial profiles of the background density $\rho_{0}(z)$ and pressure $p_{0}(z)$, and by the zero-order shear flow, etc. The aim of this paper is to present a unified theory of acoustic-gravity vortices. Besides providing a catalogue of various vortex modes, whose description is scattered in the existing literature, we also present several new solutions in the form of weakly twodimensional vortex chains. In Sect. 6, we also present a new nonlinear mechanism for the generation of shear flows in the 
atmosphere, based on the modulational instability of acoustic-gravity waves. In the strongly nonlinear phase, the latter provides the energy source for the vortex chain creation. The numerical studies also indicate the possibility of a transition to chaos.

\section{Stationary solutions}

We look for a solution of Eqs. (1) and (2) that is stationary in the reference frame travelling with a constant velocity $u$ along the $x$ axis, i.e. which is dependent only on the vertical coordinate $z$ and the coordinate $x-u t$. Using $\partial / \partial t=-u(\partial / \partial x)$ and the properties of the vector product, Eqs. (1) and (2) are readily integrated to

$\nabla^{2} \psi+u \int \frac{d z}{4 H^{2}}+q_{0} \frac{d \mathcal{F}(\psi+u z)}{d(\psi+u z)}=\mathcal{G}(\psi+u z)$,

and

$\chi=\mathcal{F}(\psi+u z)$,

where $\mathcal{F}$ and $\mathcal{G}$ are arbitrary functions of the nonlinear characteristic $\psi+u z$. We are particularly interested in nonlinear solutions that are localized, i.e. which vanish for $(x, z) \rightarrow$ $\pm \infty$. For the localized solutions, substituting the unperturbed values $\rho_{0}(z), p_{0}(z)$ and $\psi_{0}(z)$ into Eqs. (4) and (5) we can readily evaluate the asymptotic forms of the functions $\mathcal{F}$ and $\mathcal{G}$. Since they are continuous functions, their asymptotic forms are applicable along all the nonlinear characteristics $\psi(x, z)+u z=$ const . that extend to infinity in the $x, z$ plane. There may exist finite regions, usually referred to as vortex cores, in which the characteristics are closed lines, where $\mathcal{F}$ and $\mathcal{G}$ may take completely different forms, provided that the physical boundary conditions across the vortex core edge are satisfied. Obviously, the full nonlinear problem is impossible to solve in the general case, since that would involve the inversion of the unknown functions $\mathcal{F}$ and $\mathcal{G}$ along sepatrices between the regions of open and closed characteristics, whose actual shape is also not known in advance. Furthermore, such a complex problem often does not have a unique solution. The eventual stationary state (if any!) to which the system saturates in the evolution, as described by the full Eqs. (1) and (2), depends very sensitively on the initial conditions and fine details, many of which are not included in our Eqs. (4) and (5). Metaphorically, it is often said that the state of the atmosphere is determined by the flapping of a butterfly's wing at some distant past.

Naturally, analytical solutions are available only if the functions $\mathcal{F}$ and $\mathcal{G}$ have sufficiently simple forms, which are, in turn, permitted only for some specific profiles of the unperturbed quantities $\rho_{0}(z), p_{0}(z)$ and $\psi_{0}(z)$.

\section{Solitary vortices}

Solitary vortices can be constructed analytically when the functions $\mathcal{F}$ and $\mathcal{G}$ are adopted as linear, i.e.

$\mathcal{F}(\xi)=F_{0}+F_{1} \cdot \xi, \quad \mathcal{G}(\xi)=G_{0}+G_{1} \cdot \xi$, but permitting the constants $F_{0,1}$ and $G_{0,1}$ to take different values inside and outside of the vortex core. The core is chosen to be a circle with radius $r_{0}$. Although such a choice seems to be very restrictive, it represents a paradigm for vortices which are characterized by a single region of closed isolines. Due to their topological constraints, all solitary vortices (including those with more complicated expressions for $\mathcal{F}$ and $\mathcal{G}$ ) share many common features, resulting from the fact that they are produced by the nonlinearities acting within the core, leading to a linear (or weakly nonlinear) evanescent response in the outer region.

\subsection{Dipoles}

The acoustic-gravity solitary vortices that propagate relative to the surrounding fluid have the form of dipoles. They were described previously (Stenflo, 1987; Stenflo and Stepanyants, 1995). From the asymptotic considerations, it is obvious that for a non-zero vortex velocity $u$, the simple expression (6) can be satisfied in the outer region only when the unperturbed quantities $\chi_{0}(z)$ and $q_{0}(z)$ are linear functions, while $H$ is constant and $\lim _{z \rightarrow \pm \infty} \psi_{0}(z)=0$. Such atmospheric conditions may be realized only approximately for small vortices (i.e. the vortices that are much smaller than the characteristic length of the density and pressure inhomogenities).

For the linear functions $\mathcal{F}$ and $\mathcal{G}$, Eqs. (4) and (5) are readily integrated as $\chi=\chi_{0}(z)-\left(w_{g}^{2} / u\right) \psi$ and

$$
\psi=-u r_{0} \cos \theta \begin{cases}\frac{K_{1}(\mu r)}{K_{1}\left(\mu r_{0}\right)}, & r>r_{0} \\ \frac{\mu^{2}+\kappa^{2}}{\kappa^{2}} \frac{r}{r_{0}}-\frac{\mu^{2}}{\kappa^{2}} \frac{J_{1}(\kappa r)}{J_{1}\left(\kappa r_{0}\right)}, & r \leq r_{0}\end{cases}
$$

where $r=\left(x^{\prime \prime 2}+z^{2}\right)^{1 / 2}, \theta=\arctan \left(x^{\prime \prime} / z\right), x^{\prime \prime}=x-u t$, and $\mu^{2}=1 /\left(4 H^{2}\right)-\omega_{g}^{2} / u^{2}$, and where the characteristic wave number $\kappa$ is calculated from the nonlinear dispersion relation that is due to the appropriate boundary conditions at $r=r_{0}$

$$
\frac{J_{2}\left(\kappa r_{0}\right)}{\kappa J_{1}\left(\kappa r_{0}\right)}+\frac{K_{2}\left(\mu r_{0}\right)}{\mu K_{1}\left(\mu r_{0}\right)}=0
$$

Here, the constant $\omega_{g}^{2}=-(1 / u)(d / d z) \chi_{0}(z)$ is the squared Brunt-Väisälä frequency, and $J_{i}$ and $K_{i}$ are the Bessel and modified Bessel functions of the order of $i$, respectively. For typical atmospheric conditions, the vortex core radius $r_{0}$ is of the order of 10-1000 $\mathrm{m}$ in the equatorial atmosphere. The vortex velocity $u$ is close to the sound speed, except for temperature gradients corresponding to the instability threshold, $\omega_{g} \approx 0$, when the vortex speed may be much lower. For details, we refer the reader to a previous paper (Stenflo and Stepanyants, 1995).

\subsection{Tripoles}

Tripoles are known to emerge in two-dimensional incompressible flows. Stable tripolar vortices were observed in 
the Earth's atmosphere (Pingree and Le Cann, 1992) and produced in the rotating tank experiments (van Heijst and Kloosterziel, 1989). Their theoretical explanation was presented first in the context of plasma physics (Jovanović et al., 1998), and recently, they were also constructed for the acoustic-gravity mode (Jovanović et al., 2001).

Tripoles can also be analytically constructed using the simple expressions (6), but in contrast to the dipoles, they do not propagate relative to the surrounding fluid, i.e. they are carried by a zero-order flow. The solution of the basic Eqs. (4), (5), and (6), in the form of a tripole, can be obtained only in the presence of a sheared flow with a linear velocity dependence, linearly inhomogeneous inverse square length $1 / H^{2}$, and parabolic $z$-dependence of the quantities $\chi_{0}$ and $q_{0}$, i.e.

$\chi=\delta \chi-\omega_{g, 0}^{2}\left(z+\epsilon_{\chi} z^{2}\right)$,

$\psi=\delta \psi+w\left(z+\epsilon_{\psi} z^{2}\right)$

$q_{0}=z+\epsilon_{q} z^{2}$

and

$\frac{1}{4 H^{2}}=\frac{1}{4 H_{0}^{2}}\left(1+2 \epsilon_{H} z\right)$,

where the perturbations $\delta \chi$ and $\delta \psi$ are vanishing for $z \rightarrow$ $\pm \infty$, and where $\omega_{g, 0}, w, H_{0}, \epsilon_{\chi}, \epsilon_{\psi}, \epsilon_{q}$, and $\epsilon_{H}$ are constants, satisfying

$\frac{u}{w}=\frac{\epsilon_{\psi}}{\epsilon_{\chi}}-1$

and

$\left(\frac{\epsilon_{\psi}}{\epsilon_{\chi}}-1\right)\left(\frac{\epsilon_{\psi}}{\epsilon_{\chi}}-\frac{\epsilon_{H}}{\epsilon_{\chi}}\right)=4 H_{0}^{2} \frac{\omega_{g, 0}^{2}}{w^{2}}\left(1-\frac{\epsilon_{q}}{\epsilon_{\chi}}\right)$.

Under these conditions, the outer solution is readily expressed in terms of the modified Bessel functions $K_{0}, K_{2}$, of the zeroth and second order, as

$\delta \psi^{(\text {out })}=\beta_{0} K_{0}(\mu r)+\beta_{2} K_{2}(\mu r) \cos 2 \theta$.

Likewise, from the requirement that the terms which are linear in $z$ must cancel out, we obtain $F_{1}^{(\text {in })}=F_{1}^{(\text {out })}$, and the solution is, therefore,

$$
\begin{aligned}
& \delta \psi^{(i n)}=\alpha_{0} J_{0}(\kappa r)+\alpha_{2} J_{2}(\kappa r) \cos 2 \theta \\
& \quad-\frac{1}{\kappa^{2}}\left[w \epsilon_{\psi}\left(\mu^{2}+\kappa^{2}\right)\left(z^{\prime \prime 2}-z_{0}^{2}\right)+G_{0}^{(\text {out })}-G_{0}^{(\text {in })}\right] \\
& \quad+\frac{2}{\kappa^{4}} w \epsilon_{\psi}\left(\mu^{2}+\kappa^{2}\right),
\end{aligned}
$$

where $G_{0}^{(o u t)}=2 w \epsilon_{\psi}$, the e-folding number $\mu$ is determined by

$\mu^{2}=G_{1}^{(\text {out })}=\frac{1}{w \epsilon_{\psi}}\left(\frac{u \epsilon_{H}}{4 H_{0}^{2}}-\frac{\omega_{g, 0}^{2} \epsilon_{\chi} \epsilon_{q}}{w \epsilon_{\psi}}\right)$ and we have used the notations $\kappa^{2}=-G_{1}^{(i n)}, z_{0}=(w+$ $u) /\left(2 w \epsilon_{\psi}\right), z^{\prime \prime}=z+z_{0}, r=\left(x^{\prime \prime 2}+z^{\prime \prime 2}\right)^{1 / 2}$ and $\theta=$ $\arctan \left(x^{\prime \prime} / z^{\prime \prime}\right)$. Our solutions (15) and (16) must satisfy the standard physical boundary conditions across the edge of the vortex core. We require that the function $\mathcal{G}$ is continuous and that its argument $\psi+u z$ is constant at $r=r_{0}$. As a consequence, the stream function $\psi$ is also continuous across the core edge, providing the finiteness of the flow velocity. In addition, we require that the flow velocity, and consequently, the radial derivative $(\partial / \partial r) \delta \psi$, is also continuous. Eliminating the amplitudes $\alpha_{i}, \beta_{i},(i=0,2)$ from the corresponding continuity relations, we obtain the nonlinear dispersion relation

$$
\begin{aligned}
& \frac{2}{r_{0}}\left(\frac{\mu^{2}}{\kappa^{2}}+1\right)=\frac{\mu^{2}}{\kappa^{2}} \frac{\left(\partial / \partial r_{0}\right) J_{2}\left(\kappa r_{0}\right)}{J_{2}\left(\kappa r_{0}\right)} \\
& +\frac{\left(\partial / \partial r_{0}\right) K_{2}\left(\mu r_{0}\right)}{K_{2}\left(\mu r_{0}\right)} .
\end{aligned}
$$

It is worth noting that this dispersion relation is related only to the second harmonic.

\section{Vortex chains}

Vortex chains, with various forms, may arise in ordinary fluids and plasmas in the presence of sheared flows, as a result of the nonlinear saturation of the Kelvin-Helmholtz instability. They typically appear in more complicated geometries, in which the asymptotic form of the function $\mathcal{G}$ in Eq. (4) is nonlinear.

Several analytical and semi-analytical vortex chain solutions have been constructed so far from a simplified version of Eq. (4) in the form of the Liouville equation

$\nabla^{2} \psi=\mathcal{G}(\psi+u z)$.

Obviously, Eq. (19) is applicable to acoustic-gravity phenomena only if the quantities $H$ and $q_{0}$ are related through

$u \int \frac{d z}{4 H^{2}}+q_{0} \frac{d \mathcal{F}(\psi+u z)}{d(\psi+u z)}=0$.

This condition may be imposed in advance (i.e. independently of the solution for $\psi$, which is still unknown) only if $\mathcal{F}$ is a linear function on the entire $x, z$ plane. The slope of such a linear function is readily obtained from the asymptotic version of Eq. (5). Thus,

$$
\begin{aligned}
& F_{1} \equiv \frac{d \mathcal{F}(\psi+u z)}{d(\psi+u z)}=p_{0}^{-1 / \gamma}\left(z_{c r}\right) \frac{d p_{0}\left(z_{c r}\right)}{d z_{c r}} \\
& \cdot \lim _{z \rightarrow \pm \infty} \frac{1}{\psi_{0}+u z}\left[\frac{p_{0}^{1 / \gamma}\left(z_{c r}\right)}{\rho_{0}\left(z_{c r}\right)}-\frac{p_{0}^{1 / \gamma}\left(z_{0}\right)}{\rho_{0}\left(z_{0}\right)}\right]=\text { const., }
\end{aligned}
$$

where $\psi_{0}(z)$ is the unperturbed stream function, and $z_{0}$ is defined by $\psi_{0}\left(z_{0}\right)+u z_{0}=0$. Noting that in the zero-amplitude limit, the unperturbed stream function also satisfies Eq. (19), and using the quadratures, we obtain the following implicit 
relation between $\psi_{0}$ and the asymptotic form of the corresponding function $\mathcal{G}$

$z=\int^{\psi_{0}-u z} d \zeta\left[2 \int^{\zeta} d \xi \mathcal{G}(\xi)\right]^{-1 / 2}$.

\subsection{Kelvin-Stewart cat's eyes}

For the acoustic-gravity problem, the Kelvin-Stewart cat's eyes solution of the Liouville Eq. (19) was found previously (Stenflo, 1994; Shukla and Shaikh, 1998). It has the form

$\psi=-u z+A \ln \left[\cosh k z+\left(1-\beta^{-2}\right)^{1 / 2} \cos k x^{\prime \prime}\right]$,

where $A, k$ and $\beta$ are arbitrary constants. Introducing the above expression into Eq. (19), one readily obtains the corresponding expression

$\mathcal{G}(\xi)=\frac{A k^{2}}{\beta^{2}} \exp \left(-\frac{2 \xi}{A}\right)$.

The cat's eyes may be visualized as a row of identical vortices, with the typical spatial scale $2 \pi / k$, which are driven by an anti-parallel shear flow. From Eqs. (20), (21), and (23) one can see that such a solution is possible only when the unperturbed quantities $p_{0}, \rho_{0}$ and $v_{0}\left(v_{0}=-d \psi_{0} / d z\right)$ asymptotically, for large $|z|$, behave as

$v_{0}-u \sim \frac{z}{|z|}, \quad \frac{p_{0}^{1 / \gamma}}{|z| \rho_{0}} \sim$ const.

and

$\frac{\rho_{0}^{1 / 2}\left(d^{2} / d z^{2}\right) \rho_{0}^{-1 / 2}}{(d / d z) p_{0}^{1-1 / \gamma}} \sim$ const.

\subsection{Row of counter-rotating vortices}

The solution in the form of a row of counter-rotating vortices

$\psi=-u z+A \operatorname{arctanh} \frac{\kappa_{z} \cos k_{x} x}{k_{x} \cosh \kappa_{z} z}$,

is obtained from Eq. (19) when $\mathcal{G}$ is adopted as

$\mathcal{G}(\xi)=\frac{A}{4}\left(\kappa_{z}^{2}-k_{x}^{2}\right) \sinh \frac{4 \xi}{A}$,

which is possible only under the following asymptotic conditions for $|z| \rightarrow \infty$

$v_{0}=u, \quad \frac{p_{0}^{1 / \gamma}}{\rho_{0}} \sim$ const.

and

$\frac{\rho_{0}^{1 / 2}\left(d^{2} / d z^{2}\right) \rho_{0}^{-1 / 2}}{(d / d z) p_{0}^{1-1 / \gamma}} \sim$ const.

Although the nonlinear structure in the form (26) is known in the literature for the ordinary fluids and plasmas (see, e.g. Mallier and Maslowe, 1993), until now it has not been proposed in the acoustic-gravity context.

\section{Weakly two-dimensional vortex chains}

The exact analytical vortex chain solutions (23) and (24) have been obtained only for the very specific density, pressure and shear flow profiles described in Sect. 4. Naturally, nonlinear structures with similar topologies are expected to exist in a much broader variety of atmospheric configurations. As an example, in this Section, we will construct several weakly 2D (two-dimensional) chains, with $\partial \psi / \partial z \gg \partial \psi / \partial x$. However, the overall picture of the generation of vortex chains is still not clear, and additional extensive studies are necessary.

In the presence of a shear flow in the unperturbed state, whose stream function $\psi_{0}$ satisfies the Liouville equation

$\psi_{0}+u z=\mathcal{G}_{0}\left(\psi_{0}+u z\right)$,

and for the density and pressure profiles that satisfy the condition

$u \int \frac{d z}{4 H^{2}}+q_{0} \frac{d \mathcal{F}(\psi+u z)}{d(\psi+u z)}=a \cdot\left(\psi_{0}+u z\right)$,

we may rewrite our basic Eq. (4) as

$\left(\nabla^{2}-a\right)\left(\psi-\psi_{0}\right)=\mathcal{G}_{1}(\psi+u z)-\mathcal{G}_{0}\left(\psi_{0}+u z\right)$,

where $\mathcal{G}_{1}(\xi)=\mathcal{G}(\xi)-a \xi$, and $a$ is an arbitrary constant. Obviously, a localized solution $\psi \rightarrow \psi_{0}$ for $|z| \rightarrow \infty$ is obtained if the function $\mathcal{G}_{1}$ behaves asymptotically as $\lim _{\psi \rightarrow \psi_{0}}$ $\mathcal{G}_{1}(\psi+u z)=\mathcal{G}_{0}\left(\psi_{0}+u z\right)$.

Two different solutions of Eq. (32), found numerically in the context of plasma physics, can be applied here to the acoustic-gravity problem.

\subsection{Tearing and bending modes}

First, we assume that in the unperturbed state there exists a shear flow in the $x$ direction, whose velocity $\boldsymbol{e}_{x} v_{0}(z)$ is given by

$v_{0}(z)=u-A \kappa_{z} \tanh \kappa_{z} z$,

which corresponds to

$\psi_{0}=-u z+A \ln \cosh \kappa_{z} z$,

and

$\mathcal{G}_{0}(\xi)=A \kappa_{z}^{2} \exp \left(-\frac{2 \xi}{A}\right)$.

We proceed by adopting $\mathcal{G}_{1}=\mathcal{G}_{0}$ in Eq. (32), and look for a weakly 2-D solution in the form

$\delta \psi \equiv \psi-\psi_{0}=\delta \psi_{0}(z)+\delta \psi_{1}(z) \cos k_{x} x$,

with $\delta \psi_{0} \gg \delta \psi_{1}$. This permits us to rewrite Eq. (32) as two coupled equations for $\delta \psi_{0}$ and $\delta \psi_{1}$

$$
\begin{aligned}
& \left(\frac{d^{2}}{d z^{2}}-a\right) \delta \psi_{0} \\
& \quad-\frac{A \kappa_{z}^{2}}{\cosh ^{2} \kappa_{z} z}\left[\exp \left(-\frac{2 \delta \psi_{0}}{A}\right)-1\right]=0,
\end{aligned}
$$


and

$$
\begin{aligned}
& {\left[\frac{d^{2}}{d z^{2}}-k_{x}^{2}-a\right.} \\
& \left.\quad+\frac{2 \kappa_{z}^{2}}{\cosh ^{2} \kappa_{z} z} \exp \left(-\frac{2 \delta \psi_{0}}{A}\right)\right] \delta \psi_{1}=0 .
\end{aligned}
$$

Equations (32)-(35) and (37), (38) have been extensively studied (Vranješ and Jovanović, 1996, 1997). In the linear limit, Eq. (32) has a stable solution for $k_{x}<\left(\kappa_{z}^{2}-1\right)^{1 / 2}$. Conversely, a localized nonlinear solution was found from Eqs. (37) and (38) if $0 \leq \kappa_{z} \leq 1$, i.e. in the parameter range in which the linear solution is unstable. It consists of two nonlinear modes, which correspond to the even and odd solutions for $\delta \psi_{1}$. Physically, these two modes emerge due to the tearing/reconnection, and the bending of the stream lines, respectively. The wavelength of the bending mode is almost an order of magnitude larger.

We note that the asymptotic dependence of the zero-order shear flow (33) is the same as in the case of the KelvinStewart cat's eyes. The density and pressure profiles that admit this type of vortex chain are determined from Eqs. (5) and (31), with the additional requirement that $\mathcal{F}$ is a linear function which asymptotically, for $z \rightarrow \pm \infty$, yields

$\frac{p_{0}^{1 / \gamma}}{|z| \rho_{0}} \sim$ const.

and

$$
\rho_{0}^{1 / 2} \frac{d^{2}}{d z^{2}} \rho_{0}^{-1 / 2}+\frac{F_{1}}{u} \frac{(d / d z) p_{0}^{1-1 / \gamma}(z)}{\left(d / d z_{c r}\right) p_{0}^{1-1 / \gamma}\left(z_{c r}\right)} \sim \frac{z}{|z|} .
$$

From (40) one may conclude that the symmetric (i.e. the tearing/reconnection) vortex chain, (37) and (38), has the same physical nature as the Kelvin-Stewart cat's eyes, which are adjusted here to a nonsymmetric atmospheric configuration, i.e. to different values of the parameters $1 / 4 H^{2}$ and $d q_{0} / d z$ at low $(z \rightarrow-\infty)$ and high $(z \rightarrow+\infty)$ altitudes.

\subsection{Double vortex chain}

A chain of a different type is obtained when we adopt $\psi_{0}=$ $\mathcal{G}_{0}(u z)=0$, and

$\mathcal{G}_{1}(\xi)=\frac{\alpha}{2 \cosh \xi}$.

For a weakly 2-D solution (36), using (41), the basic Eq. (32) breaks down to the following two equations

$$
\left(\frac{d^{2}}{d z^{2}}-a\right) \delta \psi_{0}-\frac{\alpha}{2} \cosh ^{-1}\left(\delta \psi_{0}+u z\right)=0,
$$

and

$$
\left[\frac{d^{2}}{d z^{2}}-k_{x}^{2}-a-\frac{\alpha}{2} \frac{\sinh \left(\delta \psi_{0}+u z\right)}{\cosh ^{2}\left(\delta \psi_{0}+u z\right)}\right] \delta \psi_{1}=0 .
$$

The above system of equations was studied in Vranješ and Jovanović (1997), where a numerical solution was found in the form of two parallel vortex chains, shifted by a halfwavelength relative to each other. The asymptotic behaviour of the unperturbed quantities, for $z \rightarrow \pm \infty$, are determined from Eqs. (21) and (31) as

$\frac{p_{0}^{1 / \gamma}}{z \rho_{0}} \sim$ const.

and

$\rho_{0}^{1 / 2} \frac{d^{2}}{d z^{2}} \rho_{0}^{-1 / 2}+\frac{F_{1}}{u} \frac{(d / d z) p_{0}^{1-1 / \gamma}(z)}{\left(d / d z_{c r}\right) p_{0}^{1-1 / \gamma}\left(z_{c r}\right)} \sim$ const.

Noting that the one-dimensional component of the fluid velocity (Vranješ and Jovanović, 1997), $\delta v_{0}=-(d / d z) \delta \psi_{0}(z)$, which takes here the role of the background shear flow, features three counter-streaming flows at $z=0$ and $z= \pm a / \alpha$; this double vortex chain may be interpreted physically as a combination of two Kelvin-Stewart cat's eyes chains with opposite orientation, brought close to each other.

\section{Nonlinear generation of shear flows}

In Sects. 4 and 5, we demonstrated the existence of stationary vortex chains in atmospheric configurations, characterized by the existence of counter-streaming flows, at low and high altitudes. A plausible explanation is that such structures result from the nonlinear saturation of the Kelvin-Helmholtz instability.

It should be noted that a linear instability of acousticgravity waves also arises in the absence of shear flows, if the temperature gradient is much larger than the density gradient, i.e. for $\omega_{g}^{2}<0$ (see, e.g. Stenflo, 1990). The nonlinear three-wave interaction of such large amplitude acousticgravity waves was studied by Axelsson et al. (1996), within the framework of the weak-turbulence approximation.

In this Section, we shall study the modulation instability of short-wavelength acoustic-gravity waves, and show that it leads to the generation of large-scale horizontal shear flows. Furthermore, on the short spatial scale, we find the evidence of a transition to chaos.

We study the acoustic-gravity phenomena described by Eqs. (4) and (5) in the absence of shear flows, $\psi_{0}=0$, considering a constant characteristic length $H$, and parabolic profiles of the quantities $q_{0}(z)$ and $\chi_{0}(z)$, i.e.

$q_{0}(z)=q_{0}(0)+Q z^{2}$,

$\chi=\delta \chi+\chi_{0}(0)+\Omega z^{2}$,

where $Q$ and $\Omega$ are constants, and for simplicity, we have chosen the critical altitude to be zero $\left(z_{c r}=0\right)$. We look for a stationary solution. From Eqs. (46) and (47) the asymptotic forms of $\mathcal{F}$ and $\mathcal{G}$ are then readily found as

$\mathcal{F}(\xi)=\frac{\Omega}{u^{2}} \xi^{2}$ and $\mathcal{G}(\xi)=\frac{1}{4 H^{2}} \xi+2 \frac{\Omega Q}{u^{4}} \xi^{3}$. 


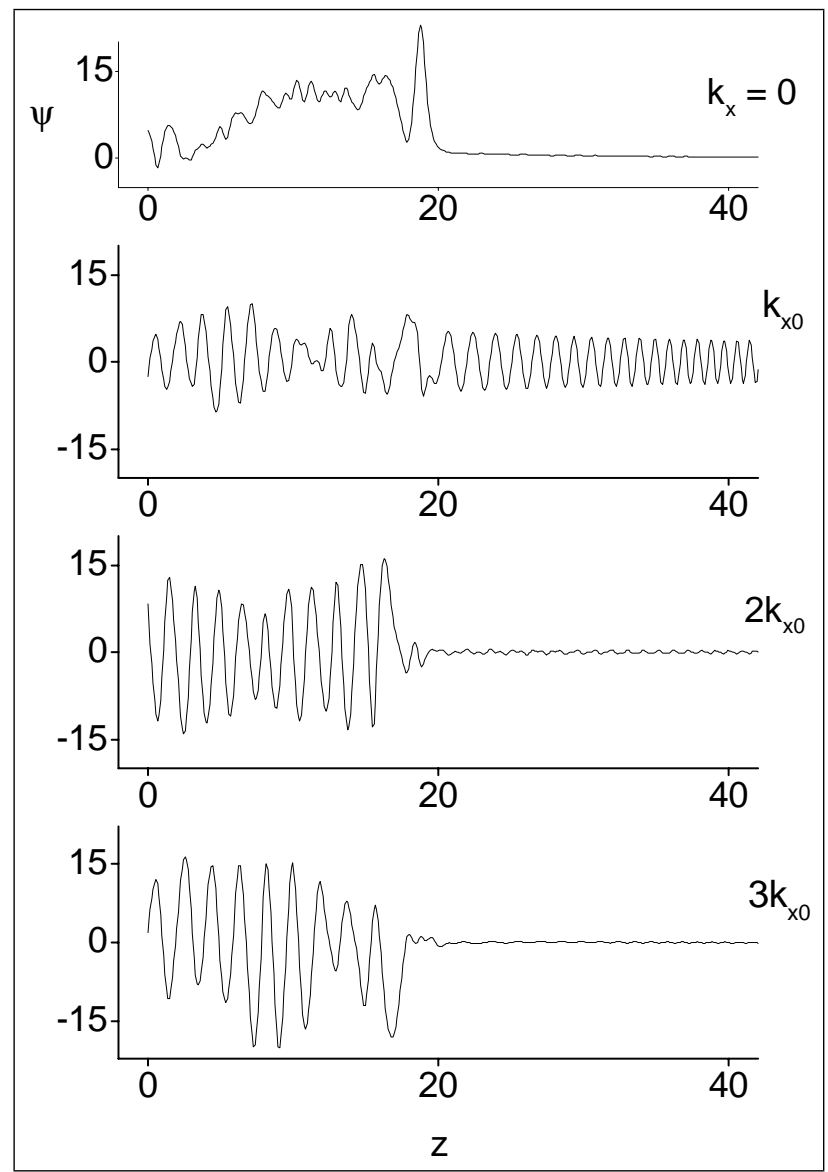

Fig. 1. The horizontal dependence of the first four $k_{x}$ Fourier components of the stream function. The top curve is the $k_{x}=0$ mode, i.e. the stream function of the zonal flow.

We use these asymptotic expressions in the entire $x, z$ plane, also those nonlinear characteristics that do not extend to infinity. Such a procedure, in the small but finite amplitude limit yields results that are equivalent to the standard coupled-mode theory. As a result, from Eq. (5), we obtain the nonlinear Schrödinger-like equation

$$
\begin{aligned}
& \left(\nabla_{\perp}^{2}-\frac{1}{4 H^{2}}\right) \psi \\
& \quad-2 \frac{\Omega Q}{u^{4}}(\psi+u z)\left[(\psi+u z)^{2}-u^{2} z^{2}\right]=0 .
\end{aligned}
$$

Here, Eq. (49) is numerically solved, and the results are displayed in Figs. 1 and 2 for the nonlinear solutions that asymptotically (at $z \rightarrow \infty$ ) coincide with a linear acousticgravity wave emitted from the critical surface $z=0$ in the horizontal direction (i.e. with $k_{x} \neq 0$ and $k_{z}=0$ ). The presence of the inhomogeneities (46) and (47) introduces a weak $z$-dependence of the effective wave number $k_{z}$ of the linear mode. With our choice of parameters, the entire atmosphere is transparent to the fundamental mode, with the turning point of the second harmonic located at $z_{t p} \approx 20$, while those of the higher $k_{x}$ harmonics are located at proportionally larger distances from $z=0$.

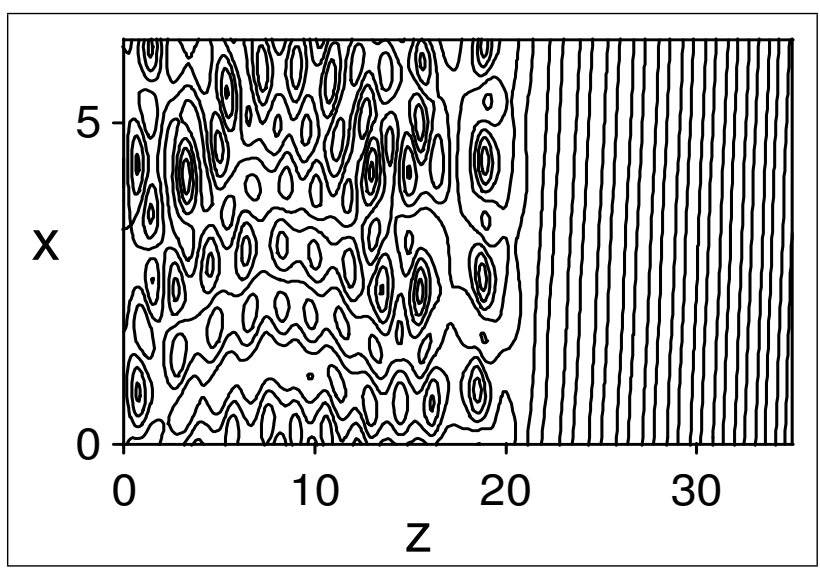

Fig. 2. The surface plot of the stream function.

Figure 1 shows that the nonlinearly generated zonal flow is spatially restricted to the critical region in which the second harmonic linear mode is evanescent, i.e. $0<z<z_{t p}$. This result indicates that the modulation instability, which is responsible for the excitation of the zonal flow in the time domain, must also involve higher-order nonlinear processes, such as the coupling with the second harmonic. We also find that the nonlinear interactions comprising evanescent Fourier modes with higher $k_{x}$ produce short-scale, nonlinear oscillations in the direction of the $z$ axis, also restricted to the region $0<z<z_{t p}$, as can be seen in Figs. 1 and 2. While the basic configuration of the zonal flow appears to be robust (see Fig. 1), the distribution of these short-scale (both in $x$ and $z$ ) quasi-modes is very sensitive to the small changes in the phase of the fundamental wave, and cannot be effectively controlled by the asymptotic boundary condition. Since they are also rather slowly converging with large $k_{x}$, their behaviour indicates the possibility of intermittency and chaos (Stenflo, 1996).

\section{Conclusions}

In this paper, we have developed a general theory of nonlinear vortex structures associated with acoustic-gravity perturbations in the Earth's atmosphere. Two classes of such nonlinear structures have been distinguished, the isolated (solitary) vortices and vortex chains. Due to their topological constraints, the solitary vortices share many common features, since they are produced by the nonlinearities acting within the core leading to a linear (or weakly nonlinear) evanescent response in the outer region. As typical examples, dipolar and tripolar solitary vortices have been presented and discussed in detail. The dipoles move relatively to the surrounding fluid, and they are much smaller than the characteristic length of the density and pressure inhomogeneities. Conversely, the tripoles are carried by the fluid, and they emerge in the presence of sheared flows and certain, rather specific, density and temperature profiles. The vortex chains have been shown to exist only in the presence of shear flows, 
while their actual form is determined by the unperturbed density, temperature and velocity profiles. Besides the classical Kelvin-Stewart cat's eyes chain, which can be realized under very restrictive conditions, a family of tearing- and bending-type vortex chains has been shown to exist under a broader class of atmospheric conditions. The mode-coupling mechanism for the nonlinear generation of shear flows in the presence of linearly unstable acoustic-gravity waves, possibly also leading to intermittency and chaos, is presented.

\section{Acknowledgement}

This work was done while one of the authors (D. J.) was visiting the Physics Department of the Ruhr University in Bochum, Germany. He is grateful for the hospitality of this institution. The work was supported in part (D. J.) by the Deutscher Akademischer Austauschdienst Grant A0119214.

\section{References}

Axelsson, P., Larsson, J., and Stenflo, L.: Nonlinear interaction between acoustic gravity waves in a rotating atmosphere, Nonlin. Proc. Geophys., 3, 216-220, 1996.

Jovanović, D., Pegoraro, F., and Rasmussen, J. J.: Tripolar shearAlfvén vortex structures, J. Plasma Phys., 60, 383-391, 1998.
Jovanović, D., Stenflo, L., and Shukla, P. K.: Acoustic gravity tripolar vortices, Phys. Lett., A 279, 70-74, 2001.

Mallier, R. and Maslowe, S. A.: A row of counter-rotating vortices, Phys. Fluids, A 5, 1074-1075, 1993.

Pingree, R. D. and Le Cann, B.: Anticyclonic eddy X91 in the southern Bay of Biscay, May 1991 to February 1992, J. Geophys. Res., 97, 14353-14 367, 1992.

Shukla, P. K. and Shaikh, A. A.: Dust-acoustic gravity vortices in a nonuniform dusty atmosphere, Phys. Scripta, T 75, 247-248, 1998.

Stenflo, L.: Acoustic solitary vortices, Phys. Fluids, 30, 3297-3299, 1987.

Stenflo, L.: Acoustic gravity vortices, Phys. Scripta, 41, 641-642, 1990.

Stenflo, L.: Acoustic gravity vortex chains, Phys. Lett., A 186, 133 134, 1994.

Stenflo, L.: Nonlinear equations for acoustic gravity waves, Phys. Lett., A 222, 378-380, 1996.

Stenflo, L. and Stepanyants, Yu. A.: Acoustic-gravity modons in the atmosphere, Ann. Geophysicae, 13, 973-975, 1995.

van Heijst, G. J. F. and Kloosterziel, R. C.: Tripolar vortices in a rotating fluid, Nature, 338, 569-571, 1989.

Vranješ, J.: Coherent structures in sheared flow of magnetized plasma, Phys. Scripta, 59, 230-234, 1999.

Vranješ, J. and Jovanović, D.: Nonlinear magnetic chains associated with plasma flow, Phys. Plasmas, 3, 2275-2279, 1996.

Vranješ, J. and Jovanović, D.: Electrostatic chains driven by nonuniform lower hybrid pump, Phys. Scripta, 55, 93-95, 1997. 